\title{
Cell-phone Based Drowsiness Monitoring and Management System
}

\author{
Yu-Te Wang, Chung-Kuan Cheng \\ Department of Computer Science and Engineering \\ University of California San Diego \\ La Jolla, USA
}

\author{
Kuan-Chih Huang, Chin-Teng Lin \\ Institute of Electrical Control Engineering \\ National Chiao-Tung University \\ Hsinchu, Taiwan
}

\author{
Yijun Wang, Tzyy-Ping Jung \\ Institute of Engineering in Medicine \\ Institute for Neural Computation \\ University of California San Diego \\ La Jolla, USA \\ jung@sccn.ucsd.edu
}

\begin{abstract}
In recent years, driving fatigue and cognitive lapses have gained increasing attentions in the fields of public security, especially regarding the safe manipulation of vehicles. Studies have explored the relation between electroencephalogram (EEG) and driving behavior and several of them further proposed effective approaches (e.g., auditory feedback) to arouse subjects from drowsiness. However, these studies were performed under laboratory-oriented configurations using tethered, ponderous EEG equipment. In real environments, the setup of bulky cognitive monitoring equipment with a long-prep time is not feasible. Therefore, this study extends previous laboratory work by developing an on-line Drowsiness Monitoring and Management (DMM) System featuring a mobile wireless drysensor EEG headgear and a cell-phone based real-time EEG processing platform. The DMM system can continuously observe EEG dynamics, deliver arousing feedback to users experiencing momentary cognitive lapses, and assess the efficacy of the feedback in near real-time.
\end{abstract}

\section{INTRODUCTION}

Driving fatigue is one of the main causes of car accidents [1-4]. Several studies [5, 6] have shown that it is feasible to accurately detect individuals' drowsiness based on their electroencephalographic (EEG) spectra in sustained-attention tasks. Recently, Lin et al. [6] also showed that arousing auditory feedback delivered to the drowsy subjects could agitate subjects' responses to the events. They further demonstrated the feasibility of estimating the efficacy of arousing feedback presented to the drowsy subjects by monitoring the changes in EEG power spectra [7, 8]. However, these results were based on laboratory-oriented EEG recordings that required uncomfortable skin preparation and cumbersome EEG equipment. This setup is impractical

This work was supported in part by the UST-UCSD International Center of Excellence in Advanced Bio-engineering sponsored by the Taiwan National Science Council I-RiCE Program under Grant Number: NSC-1002911-I-009-101, and in part by the Aiming for the Top University Plan of National Chiao Tung University, the Ministry of Education, Taiwan, under Contract 100W963. This work was also sponsored in part by US Office of Naval Research, Army Research Office (W911NF-09-1-0510) and Army Research Laboratory (W911NF-10-2-0022), and DARPA. for routine use by unconstrained, freely moving users in realworld environments.

This study proposed and implemented a portable cellphone-based, closed-loop Drowsiness Monitoring and Management (DMM) System that can continuously observe EEG dynamics, deliver arousing feedback to users experiencing momentary cognitive lapses, and assess the efficacy of the feedback in near real-time based on previous electrophysiological studies. The DMM system comprises three elements: (1) non-prep dry EEG sensors [9, 10]; (2) a small and lightweight EEG headgear developed by National Chiao-Tung University, Taiwan, featuring amplifiers, analogto-digital (ADC) converters, a TI MSP430 microprocessor, and a Bluetooth module; (3) an Android cell-phone that realizes signal-processing to monitor user's drowsiness level, delivers auditory warning feedback to the drowsy user, and assesses the efficacy of the feedback through analyzing the EEG power spectra.

\section{ELECTROPHYSILOGICAL CORRELATES OF BEHAVIORAL LAPSES}

\section{A. Driving Performance and EEG Analysis}

Previous studies have explored the behavioral changes and accompanying brain dynamics of individuals experiencing momentary cognitive lapses in sustained-attention driving tasks [11]. The simulated driving experiments were conducted in a virtual-reality environment that emulated a 90-minute continuous car drive at a fixed speed of $100 \mathrm{~km} / \mathrm{hr}$ on a fourlane freeway [11]. The car was randomly drifted away from the center of the cruising lane to mimic the consequences of a non-ideal road surface [11]. This task required subjects to compensate the car drifting by manipulating the steering to 
keep the car in the center of third cruising lane. They further investigated the effects of arousing auditory feedback on subjects' behavioral responses and brain activities [6]. Fig. 1 illustrates the experimental scenario used in the study. When subject's reaction times (RTs) to lane-departure events were over a threshold (three times longer than the mean alert RT) during driving, the system delivered a $1,750 \mathrm{~Hz}$ tone-burst to the subject in half of these drowsy trials (marked as "current trial"). A lane-departure event immediately after the current trial was labeled as the "next trial" (see Fig. 1).

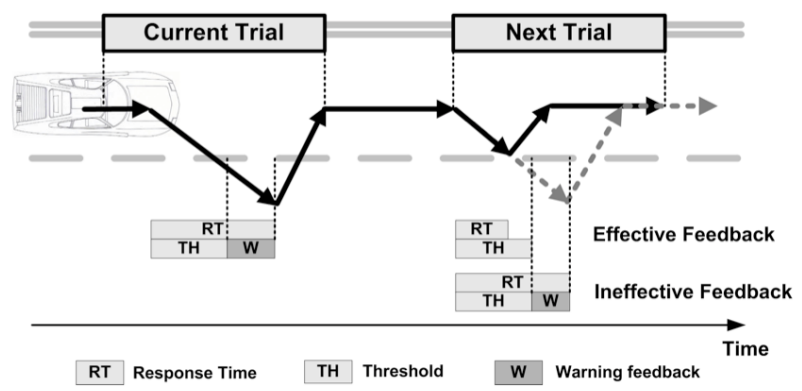

Figure 1. A top view of event-ralated lane-departure event [6].

Subsequent studies [7, 8] further reported that some trials remained non-responsive following the arousing feedback (i.e. RTs were longer than the threshold), defined as "ineffective feedback"; others had RTs shorter than two times the mean alert RT, defined as "effective feedback". The studies also showed that bilateral occipital components separated by Independent Component Analysis exhibited a broadband power increase below $25 \mathrm{~Hz}$ in drowsy trials, compared to the alert trials. For the trials in which the feedback effectively rectified subjects' behavior, the baseline spectra dropped significantly $(\mathrm{p}<0.005)$ from the current to the next trials, most prominent in the theta and alpha bands. For the ineffective trials, the power spectra were significantly higher than those of the alert trials below $25 \mathrm{~Hz}$ and did not show any appreciable change from the current to the next trials.

This study extends their work to explore the temporal dynamics of the spectral fluctuations during the transition from alertness to drowsiness and following auditory feedback. Fig. 2 shows time courses of the averaged theta- and alphaband spectra following auditory feedback delivered to the cognitively fatigued subjects, compared to those of the (black) alert and (dark blue) non-feedback trials. The results showed that:

- Both theta- and alpha- band power increased significantly from alertness (black traces) to drowsiness, as evidenced by the steady increase from $15 \mathrm{sec}$ before the arousing feedback was delivered and subjects responded to the lane-departure events (at time 0).

- Both the theta- and alpha- band power dropped significantly immediately after subject response.

- Following subject responses, the EEG power of noneffective (light blue) and non-feedback (blue) trials quickly rose from the alert baseline to the drowsy level in 5-10 seconds. The EEG power of effective (red) trials, on the other hand, remained low for $\sim 40$ seconds.

These results suggest the feasibility of continuous monitoring of the individuals' drowsiness levels by measuring the low-frequency EEG spectra. One can also assess the efficacy of auditory feedback by tracking the EEG spectra following auditory feedback. These results lay a foundation for the development of an on-line DMM system.

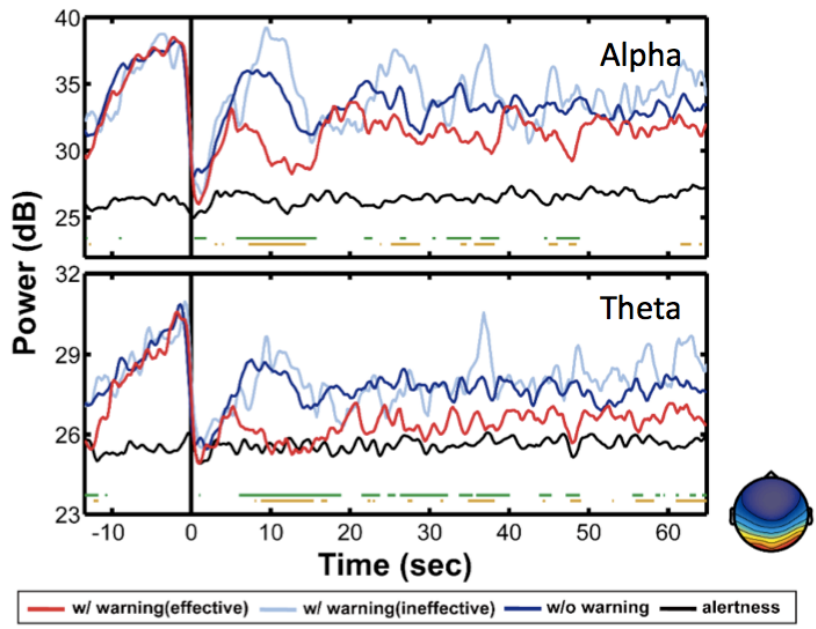

Figure 2. Time courses of the averaged alpha-band and theta-band power after auditory feedback across 11 subjects, compared to those of alert and nofeedback trials. Note the scalp map of right down corner represented the bilateral occipital component seperated by Independent Component Analysis.

\section{B. DMM System Evaluation}

Huang et al. [8] proposed a closed-loop cognitive monitoring system to augment human performance by monitoring the occipital EEG power. However, the proposed system here was not implemented in that study. Furthermore, Huang's study was performed in a laboratory-oriented setting using wired, high-density, ponderous EEG equipment. In real environments, the setup of bulky cognitive monitoring equipment with a long-prep time is not feasible. This study developed an on-line DMM system featuring a mobile and wireless dry-sensor EEG headgear and a cell-phone based, real-time EEG platform that can assess the cognitive states of the subjects in near real-time.

\section{IMPLEMENTATION OF AN ON-LINE AND MOBILE DMM SYSTEM}

\section{A. System Hardware Design}

Fig. 3 shows the system diagram of the portable and mobile DMM system. It depicts two major components of the proposed system: (1) a mobile and wireless EEG headgear and (2) a cell-phone based DMM platform.

The mobile and wireless EEG headgear is a 4-channel lightweight portable bio-signal acquisition device. It consists of five parts: (1) a TI MSP430 microprocessor, (2) a preamplifier and battery charger circuit, (3) a 24-bit ADC, (4) a Bluetooth module, and (5) dry spring-loaded EEG sensors [9, 
10]. The sensors go through the hair and measure EEG signals from the occipital area without requiring skin preparation and gel application to ensure good electrical conductivity between the sensors and skin. The MSP 430 microprocessor is the major component for controlling all passive elements on the circuit. When a subject puts the Mobile and Wireless EEG headgear over the occipital area and turns on the power, the EEG data are amplified by pre-amplifiers, digitized by ADC, and then transmitted by the Bluetooth module automatically. The sampling rate can be programed to 128,256 or $512 \mathrm{~Hz}$. The digitized 4-channel EEG data are sequentially transmitted to the cell-phone based EEG platform to perform DMM as well as deliver auditory arousing feedback. Table 1 lists the detailed specifications of the mobile and wireless EEG headgear.

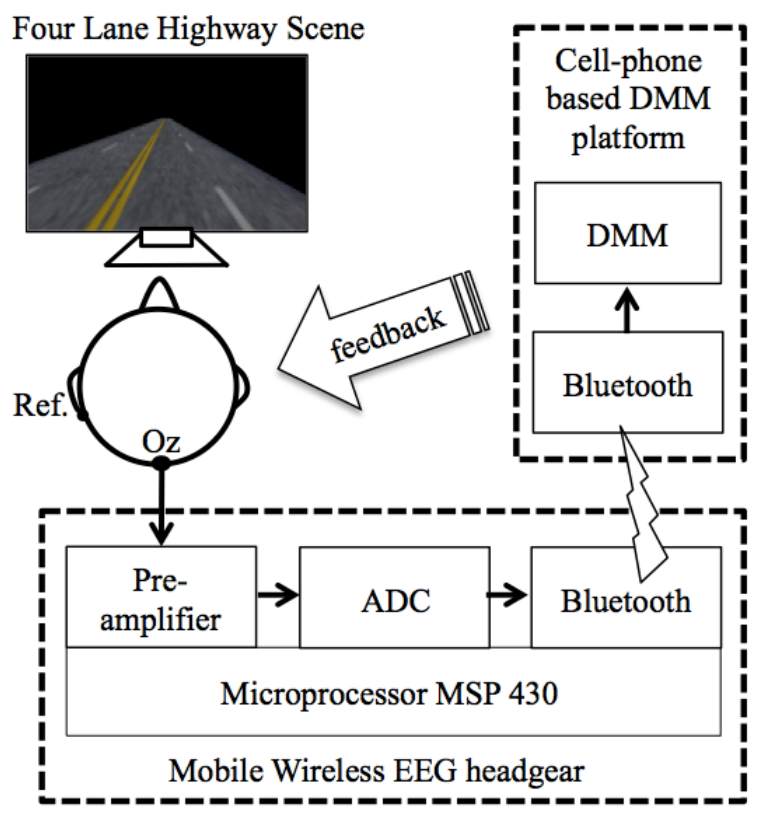

Figure 3. The system diagram of the proposed DMM System.

The DMM system was implemented as an App running on a Samsung Galaxy S2 cell-phone. The phone was equipped a Bluetooth module, a 1.2Ghz dual-core CPU (Cortex A9), 1GB RAM, and Android V2.3.4 (Gingerbread) OS. When the App was launched, it first searched for the Mobile and Wireless EEG headgear and created a connection automatically once it has been found. Then, the baseline was calculated for the operation of the DMM system. After, the acquired 4-channel EEG raw data were processed and analyzed in near real-time to perform DMM. The received data could be stored onto the internal or external memory card. Fig. 4 shows the processing sequence of the App.

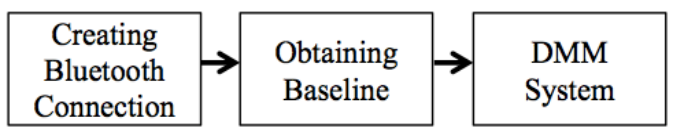

Figure 4. The function blocks of the cell-phone based DMM platform.
TABLE I. THE SPECIFICATIONS FOR MOBILE WIRELESS EEG UNIT.

\begin{tabular}{|l|l|}
\hline \multicolumn{1}{|c|}{ Specification } & \multicolumn{1}{c|}{ Value } \\
\hline Analog digital converter & 24 bits \\
\hline Digital resolution: & $0.29 \mathrm{uV}$ \\
\hline Sampling rate: & $128 / 256 / 512 \mathrm{~Hz}$ \\
\hline Circuit size $\left(\mathrm{L}^{*} \mathrm{~W} * \mathrm{H}\right):$ & $45 * 35^{*} 4 \mathrm{~mm}$ \\
\hline Weight & $105 \mathrm{~g}$ \\
\hline Power consumption & $>12 \mathrm{Hr}(3.7 \mathrm{v})$ \\
\hline
\end{tabular}

\section{B. EEG Data Processing}

The power spectrum was obtained by applying Fast Fourier Transform (FFT) to the continuous 4-channel EEG data using a 256-point sliding window with a $1 \mathrm{sec}$ step. Alpha-band and theta-band power spectra were both considered as key indices to identify the subject's drowsiness and unresponsiveness to auditory arousing feedback.

\section{System Software Design}

The DMM system implements a real-time arousing feedback algorithm that can continuously monitor a subject's cognitive state. The system consists of two states: (1) driving performance monitoring state and (2) feedback efficacy assessment state. Fig. 5 shows the finite state diagram of the DMM system. At the beginning, the program enters the driving performance monitoring state. In this state, the DMM system monitors the subject's cognitive state by analyzing the power of theta and alpha bands. Once the EEG power is $6 \mathrm{~dB}$ above the baseline, the DMM system will deliver an auditory feedback and enter the feedback efficacy assessment state. In the meantime, the current powers of alpha and theta bands are stored temporarily for the comparison in the next state. In the feedback efficacy assessment state, the arousing auditory feedback is delivered every second until the power of alpha and theta bands drop $6 \mathrm{~dB}$ from the drowsy power value obtained from the driving performance monitoring state. Fig. 6 shows the wearable DMM system on a subject.

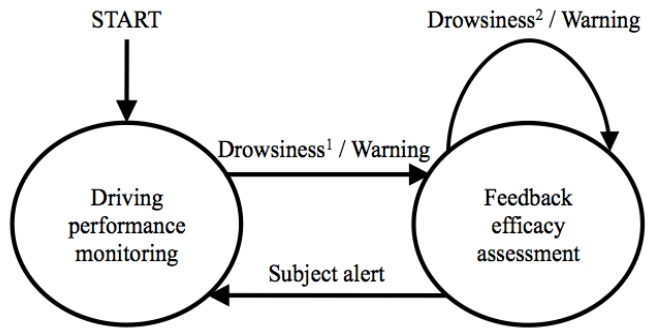

Figure 5. The state diagram of DMM system. Note that drowsiness represents that the power of subject's cognitive level is $6 \mathrm{~dB}$ over baseline. Drowsiness ${ }^{2}$ represents that the power of subject's cognitive level has not dropped $6 \mathrm{~dB}$ from the drowsy state. 


\section{DISCUSSIONS AND CONCLUSIONS}

This study first explored the electrophysiological correlates of cognitive lapses and then developed a DMM system featuring a mobile and wireless EEG headgear with an on-line and real-time signal-processing platform. Most importantly, the signal-processing platform has been moved from a PC or laptop to a commercial Android cell-phone without losing performance. The system's portability and wearability considerably improve the usability and practicality of the DMM system over traditional laboratory-oriented EEG-based brain-computer interface designs.

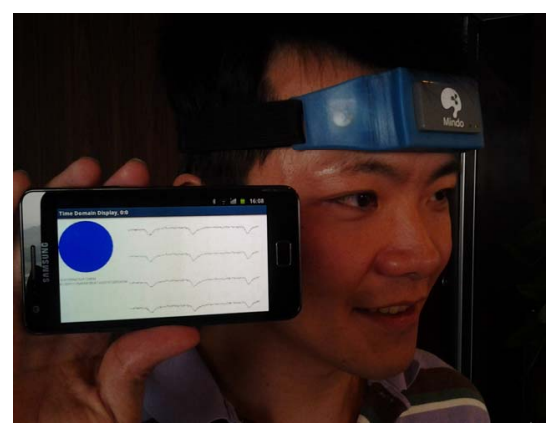

Figure 6. A subject wearing a mobile and wireless EEG unit and holding a Samsung Galaxy s2 cell-phone based DMM system.

\section{ACKNOWLEDGMENT}

Melody Jung is appreciated for her editorial assistance.

\section{REFERENCES}

[1] S. Makeig and M. Inlow, "Lapses in alertness: coherence of fluctuations in performance and EEG spectrum,"
Electroencephalography and Clinical Neurophysiology, 86: 23-35, Jan 1993.

[2] S. Makeig and T. P. Jung, "Changes in alertness are a principal component of variance in the EEG spectrum," Neuroreport, 7: 213-6, 1995.

[3] P. Philip, J. Taillard, M. A. Quera-Salva, B. Bioulac, and T. Akerstedt, "Simple reaction time, duration of driving and sleep deprivation in young versus old automobile drivers," Journal of Sleep Research, 8: 914, 1999.

[4] A. Campagne, T. Pebayle, and A. Muzet, "Correlation between driving errors and vigilance level: influence of the driver's age," Physiology \& behavior, 80: 515-24, 2004.

[5] S. Makeig and T. P. Jung, "Tonic, phasic, and transient EEG correlates of auditory awareness in drowsiness," Brain Res Cogn Brain Res, 4: $15-25,1996$.

[6] C. T. Lin, K. C. Huang, C. F. Chao, J. A. Chen, T. W. Chiu, L. W. Ko, and T. P. Jung, "Tonic and phasic EEG and behavioral changes induced by arousing feedback," Neuroimage, vol. 52, pp. 633-642, 2010.

[7] T. P. Jung, K. C. Huang, C. H. Chuang, J. A. Chen, L. W. Ko, T. W. Chiu and C. T. Lin, "Arousing feedback rectifies lapse in performance and corresponding EEG power spectrum," Proceeding of the IEEE EMBC 2010, pp. 1792 - 1795, 2010.

[8] K.C. Huang, T. P. Jung, C. H. Chuang, L. W. Ko, and C. T. Lin, Preventing lapse in performance using a drowsiness monitoring and management system. Proceeding of the IEEE EMBC 2012, in press.

[9] L. D. Liao, I. J. Wang, S. F. Chen, J. Y. Chang, and C. T. Lin, "Design, fabrication and experimental validation of a novel dry-contact sensor for measuring electroencephalography signals without skin preparation," Sensors, vol. 11, pp. 5819-5834, 2011.

[10] C. T. Lin, L. D. Liao, Y. H. Liu, I. J. Wang, B. S. Lin, and J. Y. Chang, "Novel dry polymer foam electrodes for long-term EEG measurement," IEEE Transactions on Biomedical Engineering, vol. 58, pp. 1200-1207, 2011.

[11] R. S. Huang, T. P. Jung, and S. Makeig, "Event-related brain dynamics in continuous sustained-attention tasks." In D.D. Schmorrow, L.M. Reeves (Eds.): Augmented Cognition, HCII 2007, LNAI 4565, 65-74, 2007. 